\title{
Simulación en Aspen de la combustión de mezclas diesel-biodiesel
}

\section{Aspen Simulation of Diesel-Biodiesel Blends Combustion}

\author{
Pérez-Sánchez Armando \\ Instituto de Ingeniería \\ Universidad Autónoma de Baja California \\ Correo: armand_perez@hotmail.es \\ Montero-Alpírez Gisela \\ Instituto de Ingeniería \\ Universidad Autónoma de Baja California \\ Correo:gmontero@uabc.edu.mx \\ Ayala-Bautista Ramón \\ Instituto de Ingeniería \\ Universidad Autónoma de Baja California \\ Correo:iq.ramonayala@gmail.com
}

\author{
Coronado-Ortega Marcos Alberto \\ Instituto de Ingeniería \\ Universidad Autónoma de Baja California \\ Correo:coronado.marcos@gmail.com \\ García-González Conrado \\ Academia de Química y Bioquímica \\ Instituto Tecnológico de Mexicali \\ Correo:cnrdgarciag@gmail.com
Campbell- Ramírez Héctor Enrique
Instituto de Ingeniería
Universidad Autónoma de Baja California
Correo:hcampbellr@gmail.com

Información del artículo: recibido: junio de 2013, aceptado: octubre de 2013

\section{Resumen}

El biodiesel es un combustible producido mediante la transesterificación de aceites vegetales o grasas animales, que en la actualidad está adquiriendo mayor atención como sustituto del diesel. Representa una oportunidad para disminuir las emisiones de $\mathrm{CO}_{2}, \mathrm{SO}_{2}, \mathrm{CO}, \mathrm{HC}, \mathrm{HAP}$ y $\mathrm{MP}$, además de contribuir a la diversificación de combustibles en la matriz energética de México. En el presente trabajo se muestran los resultados de la simulación de un proceso de combustión, tomando como referencia las especificaciones de un motor KUBOTA D600-B, que opera con mezclas diesel-biodiesel en diversas proporciones. Las propiedades fisicoquímicas de los compuestos y las condiciones de operación de equipos se desarrollaron utilizando el simulador Aspen ${ }^{\circledR}$ como información complementaria. Se consideraron los principales aspectos de las condiciones de operación del motor tales como: relación diesel-biodiesel, mezcla aire/combustible, temperatura de los gases de combustión y carga térmica. Las especificaciones fisicoquímicas del diesel se tomaron de los reportes de PEMEX y SENER. Los ésteres metílicos correspondientes a la transesterificación de los ácidos grasos que conforman el aceite de higuerilla fueron considerados como moléculas representativas del biodiesel y obtenidos a partir de un análisis cromatográfico. Los resultados obtenidos incluyen las emisiones de $\mathrm{CO}_{2}$, vapor de agua, eficiencia de combustión, potencia y poder calorífico inferior (PCI) de los combustibles.

\section{Descriptores:}

- Aspen

- biodiesel

- combustión

- emisiones

- energía renovable

- simulación 


\begin{abstract}
Biodiesel is a fuel produced by transesterification of vegetable oils or animal fats, which currently is gaining attention as a diesel substitute. It represents an opportunity to reduce $\mathrm{CO}_{2}, \mathrm{SO}_{2}, \mathrm{CO}, \mathrm{HC}, \mathrm{PAH}$ and PM emissions and contributes to the diversification of fuels in Mexico's energetic matrix. The results of the simulation of the combustion process are presented in this paper with reference to an engine specification KUBOTA D600-B, operated with diesel-biodiesel blends. The physicochemical properties of the compounds and the operating conditions of equipment were developed using the simulator Aspen ${ }^{\circledR}$ and supplementary information. The main aspects of the engine working conditions were considered such as diesel-biodiesel ratio, air/fuel mixture, temperature of the combustion gases and heat load. Diesel physicochemical specifications were taken from reports of PEMEX and SENER. Methyl esters corresponding to the transesterification of fatty acids that comprise castor oil were regarded as representative molecules of biodiesel obtained from chromatographic analysis. The results include $\mathrm{CO}_{2}$, water vapor, combustion efficiency, power and lower calorific value of fuels.
\end{abstract}

\section{Introducción}

En los últimos años la producción y consumo de biodiesel a nivel internacional, ha adquirido un mayor auge como resultado del encarecimiento del petróleo y sus derivados, los altos índices de emisiones contaminantes, así como la necesidad de impulsar el compromiso con el desarrollo sustentable. La importancia del biodiesel se ha señalado en obras recientes (Graboski y McCormick, 1998; Canacki, 2007; Lin et al., 2006; Rakopoulos et al., 2006; Altin et al., 2001; Iscan y Aydin, 2012), como un combustible alternativo para el motor diesel. El biodiesel es una mezcla de ésteres metílicos de ácidos grasos que se obtiene de fuentes renovables, como aceites vegetales o grasas animales, a través de un proceso de transesterificación. Esta reacción puede ser catalizada por álcalis, ácidos (Canacki y Gerpen, 1999; Vyas et al., 2010) o enzimas (Nielsen et al., 2008). Se han realizado varios estudios utilizando diferentes aceites como materia prima, distintos alcoholes, al igual que una variedad de catalizadores, incluyendo catalizadores homogéneos como el hidróxido de sodio, hidróxido de potasio y ácido sulfúrico, y catalizadores heterogéneos como las lipasas (Nielsen et al., 2008; Boey et al., 2009; Refaat, 2011).

La razón principal por la cual es necesario llevar a cabo este proceso de conversión, de aceites a biodiesel, es reducir su viscosidad, obteniendo propiedades similares a las del diesel. Entre las ventajas principales del biodiesel destacan su origen renovable, biodegradabilidad, menores emisiones de $\mathrm{CO}_{2}$ y en ocasiones emisiones nulas de $\mathrm{SO}_{x}$. Además, presenta buenas características lubricantes y tiene un punto de inflamación su- perior al del diesel. Por otra parte, se puede encontrar en la literatura la mención de algunos problemas técnicos relacionados con su uso, por ejemplo, el aumento de emisiones de $\mathrm{NO}_{x^{\prime}}$ problemas de desempeño como: torque, potencia, consumo específico de combustible y corrosión en el sistema de combustión. Las propiedades que presentan la mayor variación entre biodiesel y diesel son: la densidad, el número de cetano, el poder calorífico y, en mayor proporción, la viscosidad del combustible (Lin et al., 2006; Rakopoulos, 2006; Scarpa y Guerci, 1982). Diferentes estudios en el tema, explican la variación de los parámetros de desempeño, por la presencia de oxígeno en la composición del combustible, la cual incide en la disminución del poder calorífico y con este, la disponibilidad de energía. Sin embargo, se encuentra que el biodiesel producido a partir de diferentes fuentes (algodón, soya, palma, girasol, etcétera) (Altin et al., 2001; Iscan y Aydin, 2012), no presenta cambios significativos en su poder calorífico.

El propósito de un motor de combustión interna es la producción de energía mecánica a partir de la energía química almacenada en el combustible. En los motores de combustión interna, la energía se libera mediante el encendido y la oxidación del combustible dentro del motor, a diferencia de los de combustión externa, por ejemplo, un horno industrial o una caldera. El fluido de trabajo cambia su composición química después de la combustión y es el encargado de transferir el trabajo al pistón, y este último al resto de los componentes de la cadena cinemática, hasta llegar finalmente a las ruedas impulsoras del movimiento.

En el presente trabajo, se tomaron una serie de consideraciones relevantes para simular la etapa del proce- 
so de combustión en Aspen Plus®, utilizando combustible diesel y biodiesel, así como mezclas de ambos (B5, B10, B20 y B50). Se efectuó la estimación de las emisiones asociadas a la combustión de diesel y sus mezclas diesel-biodiesel. Asimismo, se determinaron las propiedades fisicoquímicas de un biodiesel que se definió como componente de usuario, a partir de una mezcla de ésteres metílicos, con el objetivo de simular un combustible lo más apegado a la realidad. Simulaciones desarrolladas previamente en otras investigaciones, solamente han utilizado una molécula representativa del biodiesel (Benavides et al., 1982).

\section{Aceite de higuerilla}

La higuerilla (Ricinus communis L.) es una planta oleaginosa que se cultiva de manera intensiva, principalmente para extraer el aceite de la semilla y procesarlo para obtener los ésteres metílicos correspondientes a los ácidos grasos del aceite, esto es biodiesel. Este cultivo no requiere terrenos fértiles, ya que prolifera en suelos áridos, pobres en nutrientes, con altos niveles de radiación solar y baja precipitación pluvial. Aunque el origen de la higuerilla no se ha determinado, se especula que es originaria de África, India o China (Scarpa y Guerci, 1982). Es una de las plantas más comunes en México y se encuentra en varios estados, entre los cuales se incluye Baja California. Por otra parte, se está posicionando como un cultivo energético promisorio para la producción de biodiesel debido a que no es comestible, a su alto contenido de aceite, su adaptabilidad a suelos y condiciones climáticas diversas.

Actualmente en el Instituto Nacional de Investigaciones Forestales, Agrícolas y Pecuarias (INIFAP) se realizan proyectos para la tecnificación y mejoramiento genético de la higuerilla, con el objetivo de desarrollar nuevos genotipos que ofrezcan mayores rendimientos de aceite y mayor resistencia a climas extremos como los que se presentan en la región (Scarpa y Guerci, 1982; INIFAP, 2009).

\section{Ciclo diesel}

El ciclo diesel fue desarrollado por Rudolf Diesel a principios del siglo XX. Consiste en cuatro etapas fundamentales: admisión, compresión, expansión y escape, como se ilustra en la figura 1. El ciclo inicia con la apertura de la válvula de admisión para succionar aire y llenar el cilindro. Enseguida, la válvula de admisión se cierra llevando el pistón al punto muerto inferior. El pistón se eleva, comprimiendo y calentando el aire hasta llevarlo a una temperatura de ignición. Posterior- mente, la inyección de combustible empieza cerca del punto muerto superior en la carrera de compresión y continúa durante aproximadamente $40^{\circ}$ de rotación del cigüeñal. El combustible se inflama y, debido a la expansión, el pistón es impulsado hacia abajo. Por último, la válvula de escape se abre y el pistón se eleva en la carrera de escape, purgando el cilindro de los gases de combustión. Cuando el pistón regresa al punto muerto superior, se completan los cuatro ciclos de carrera (Heywood, 1988).

\section{Emisiones de combustión}

Una de las principales ventajas de utilizar biodiesel como combustible sustituto del diesel en motores de combustión interna, es la oportunidad de reducir las emisiones contaminantes $\mathrm{HC}, \mathrm{CO}, \mathrm{CO}_{2}, \mathrm{SO}_{x^{\prime}} \mathrm{HAP}$ (hidrocarburos aromáticos policíclicos) y MP (material particulado), así como los efectos negativos causados al ambiente (gases de efecto invernadero) y en la salud humana (enfermedades crónico-respiratorias, asma entre otras); dado que algunos de los contaminantes generados por la combustión del diesel como el MP y los HC son tóxicos y carcinógenos (National Renewable Energy Laboratory, 2009). Numerosos estudios han mostrado los efectos positivos del biodiesel en la calidad del aire.

Por otra parte, cuando el biodiesel es de origen vegetal las emisiones de $\mathrm{SO}_{x}$ son prácticamente nulas. Esto representa una ventaja competitiva con respecto al diesel que contiene azufre. En lo que concierne a las emisiones de $\mathrm{CO}_{2}$ generadas por la combustión del biodiesel, estas se reintegran al ciclo de $\mathrm{CO}_{2}$ que utilizan las plantas oleaginosas para su crecimiento y desarro1lo. Este balance no resulta neutro, debido a que se requiere una demanda energética para la siembra y cosecha del cultivo, lo que implica la quema de combustibles fósiles para efectuarlo y emisiones asociadas como se observa en la tabla 1.

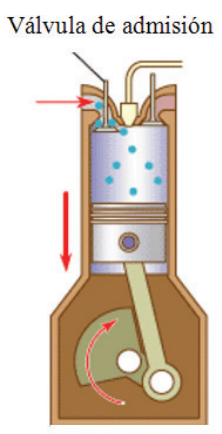

Admisión

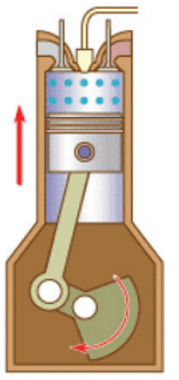

Compresión

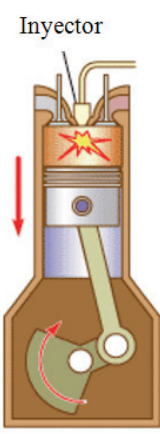

Expansión

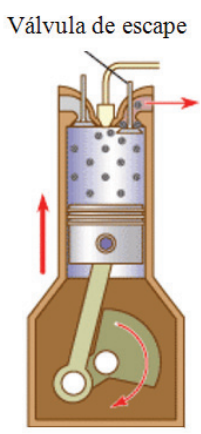

Escape
Figura 1. Ciclo diesel 
Tabla 1. Comparativo de emisiones del biodiesel (National Renewable Energy Laboratory, 2009)

\begin{tabular}{lll}
\hline \multicolumn{1}{c}{ Tipo de Emisiones } & B20 & B100 \\
\hline Hidrocarburos no quemados & $-20 \%$ & $-67 \%$ \\
$\mathrm{CO}$ & $-12 \%$ & $-48 \%$ \\
$\mathrm{CO}_{2}$ & $-16 \%$ & $-79 \%$ \\
$\mathrm{PM}$ & $-12 \%$ & $-47 \%$ \\
$\mathrm{NO}_{x}$ & $+2 \%$ & $+10 \%$ \\
$\mathrm{SO}_{x}$ & $-20 \%$ & $-100 \%$ \\
$\mathrm{Hidrocarburos}$ Aromáticos & $-13 \%$ & $-80 \%$ \\
Policíclicos (HAP) & $-50 \%$ & $-90 \%$ \\
Nitro- HAP & & \\
\hline
\end{tabular}

El uso de los combustibles oxigenados, como el biodiesel, ha demostrado ser una forma efectiva para reducir las emisiones de hollín en motores diesel (Nielsen et al., 2008; Boey et al., 2009). Los combustibles oxigenados reducen la formación de hollín mediante la creación de enlaces carbono-oxígeno y disminuyen el contenido de compuestos aromáticos en comparación con los combustibles derivados del petróleo.

En México, existe una Ley de promoción y desarrollo de los bioenergéticos, que entró en vigor en febrero de 2008. Tiene como objetivo la promoción y desarrollo de los bioenergéticos, sin poner en riesgo la seguridad y soberanía alimentaria del país, por el uso de materia prima considerada como alimento; así como procurar la reducción de emisiones contaminantes a la atmósfera y gases de efecto de invernadero, utilizando para ello los instrumentos internacionales contenidos en los tratados que México ha firmado.

La normatividad oficial aún no contempla las emisiones provenientes de la combustión de biocombustibles. Sin embargo, la norma NOM-076-SEMARNAT1995, establece los niveles máximos permisibles de emisión de hidrocarburos no quemados, monóxido de carbono y óxidos de nitrógeno provenientes del escape, así como de hidrocarburos evaporativos provenientes del sistema del combustible que usan gasolina, gas LP, gas natural y otros combustibles alternos.

\section{Materiales y métodos}

\section{Modelo de simulación}

En el presente trabajo se utilizó el Software Aspen ${ }$, herramienta que permitió simular el proceso de combustión del ciclo diesel, considerando las condiciones de operación de un motor diesel de 3 pistones, mostradas en la tabla 2.
Tabla 2. Descripciones técnicas del motor tomado como referencia

\begin{tabular}{ll}
\hline \multicolumn{1}{c}{ Especificación } & \multicolumn{1}{c}{ Textron KUBOTA D600-B } \\
\hline Tipo & Diesel, inyección directa \\
Alimentación & Presión atmosférica \\
Desplazamiento & $600 \mathrm{cc}$ \\
Configuración & 3 cilindros en línea 4 ciclos \\
Diámetro X carrera & $64 \times 62.2(\mathrm{~mm})$ \\
Relación de compresión & 18 \\
Consumo de combustible & $0.48 \mathrm{~kg} / \mathrm{h}$ \\
Potencia & $16.5 \mathrm{HP}$ \\
\hline
\end{tabular}

En la simulación se realizaron corridas con diesel y mezclas diesel-biodiesel B5, B10, B20 y B50, así como B100 (donde el número consecutivo a la letra B denota el porcentaje de biodiesel presente) en las cuales se utilizó una relación masa-masa de aire-combustible de 1:15 (Workshop, manual diesel engine Kubota). Como información adicional, se ingresaron al simulador datos de temperatura de gases de combustión que se midieron con una cámara termográfica Fluke Ti30, con rango de temperatura de $-15^{\circ} \mathrm{C}$ a $255^{\circ} \mathrm{C}$, como se muestra en la figura 2.

La lectura de las temperaturas se tomó en la salida de los gases de escape del bloque del motor KUBOTA D600-B. Se consideró una presión dentro de la cámara de combustión de $4.6 \mathrm{MPa}$, basada en el manual de Taller de Motores Diesel KUBOTA.

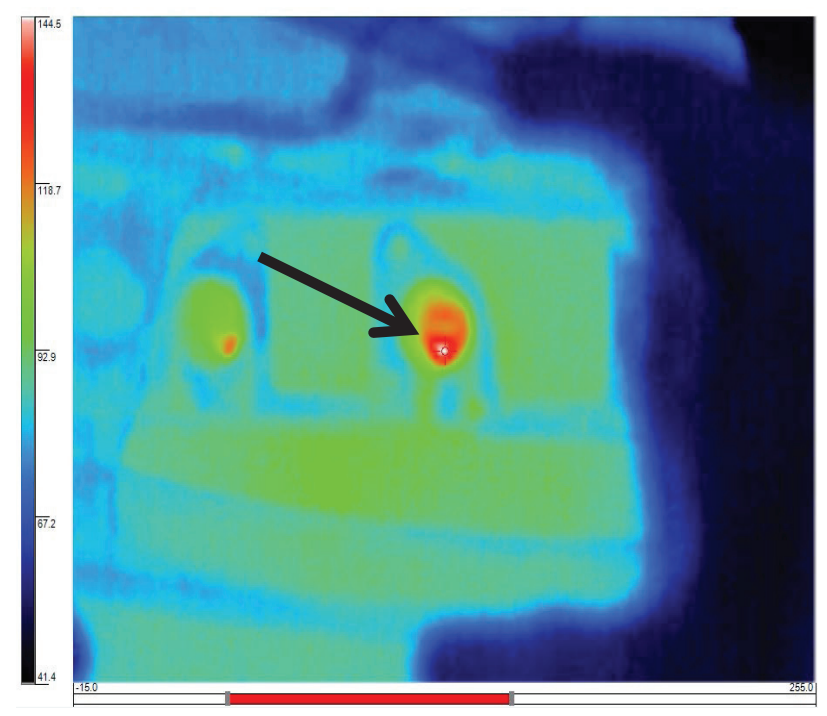

Figura 2. Imagen térmica tomada en la salida de los gases de combustión del block del Motor 
Bases de la simulación

Para realizar la simulación del proceso de combustión de las mezclas de combustibles, como se puede observar en la figura 3 , se definieron las corrientes de entrada al mezclador, para obtener la corriente de salida FUELMIX. En la corriente AIR, se especificó la mezcla en porcentaje de aire estándar compuesta por $21 \%$ de $\mathrm{O}_{2}$ y $79 \%$ de $\mathrm{N}_{2}$. Cabe mencionar que se consideró un exceso de aire para asegurar una combustión completa.

En lo que corresponde al proceso de combustión, se utilizó un modelo de reactor ideal. Dicho reactor es estequiométrico, por lo que se especificaron las reacciones de combustión para cada una de las mezclas de combustibles. A la salida del reactor la corriente de proceso denominada GASEMISN está conformada por los productos de combustión: $\mathrm{CO}_{2}, \mathrm{NO}_{2}$ y $\mathrm{H}_{2} \mathrm{O}$. Las condiciones de entrada de cada uno de los combustibles y sus componentes se muestran en las tablas 3 y 4 .

Tabla 3. Condiciones de las corrientes de alimentación del diesel y biodiesel utilizadas en la simulación

\begin{tabular}{ccccc}
\hline \multirow{2}{*}{$\begin{array}{c}\text { Flujo másico } \\
(\mathrm{kg} / \mathrm{h})\end{array}$} & Aire & Diesel & Aire & Biodiesel \\
\cline { 2 - 5 } & 0 & 0 & 0 & 0.0193 \\
Oleato de Metilo & 0 & 0 & 0 & 0.02143 \\
Linoleato de Metilo & 0 & 0 & 0 & 0.444055 \\
Ricinoleato de Metilo & 0 & 0 & 0 & $5.43 \mathrm{E}-03$ \\
Palmitato de Metilo & 0 & 0 & 0 & $5.43 \mathrm{E}-03$ \\
Estearato de Metilo & 0 & 0 & 0 & $2.18 \mathrm{E}-03$ \\
Linolenato de Metilo & 0 & 0 & 0 & $2.18 \mathrm{E}-03$ \\
Eicoseonato de Metilo & 0 & 0 & 0 & 0 \\
Diesel & 0 & 0.5 & 0 & 0 \\
Oxígeno & 12.98367 & 0 & 10.24863 & 0 \\
Nitrógeno & 48.84333 & 0 & 38.55437 & 0 \\
\hline
\end{tabular}

\section{Composición de combustibles}

\section{Biodiesel}

Para representar al biodiesel en el simulador, se utilizaron las propiedades físicas, químicas y termodinámicas de los ésteres metílicos de ácidos grasos correspondientes al aceite de higuerilla. La proporción de dichos ésteres metílicos se basó en los resultados de un análisis cromatográfico cuyos resultados se detallan en la tabla 5 (Refaat, 2011).

\section{Diesel}

El n-hexadecano se consideró como molécula representativa del diesel debido a que se encuentra en mayor proporción en el diesel (Cedeño et al., 2005) y posee un PCI de $43.94 \mathrm{MJ} / \mathrm{kg}$, muy semejante al reportado para el diesel mexicano con un valor de $44.04 \mathrm{MJ} / \mathrm{kg}$ (SENER, 2009).

\section{Método termodinámico}

La selección del método termodinámico apropiado para efectuar la simulación de procesos, se realizó considerando cuatro factores: la naturaleza de las propiedades de interés, la composición de las mezclas, rangos de presión y temperatura; así como la disponibilidad de parámetros. El método base elegido fue SR-POLAR (Carlson, 1996).

\section{Reacciones Químicas}

Las reacciones químicas consideradas en la combustión de los ésteres metílicos (biodiesel), diesel y sus diferentes mezclas (B5, B10, B20, B50) que fueron ingresadas al simulador, se destacan en la tabla 6.

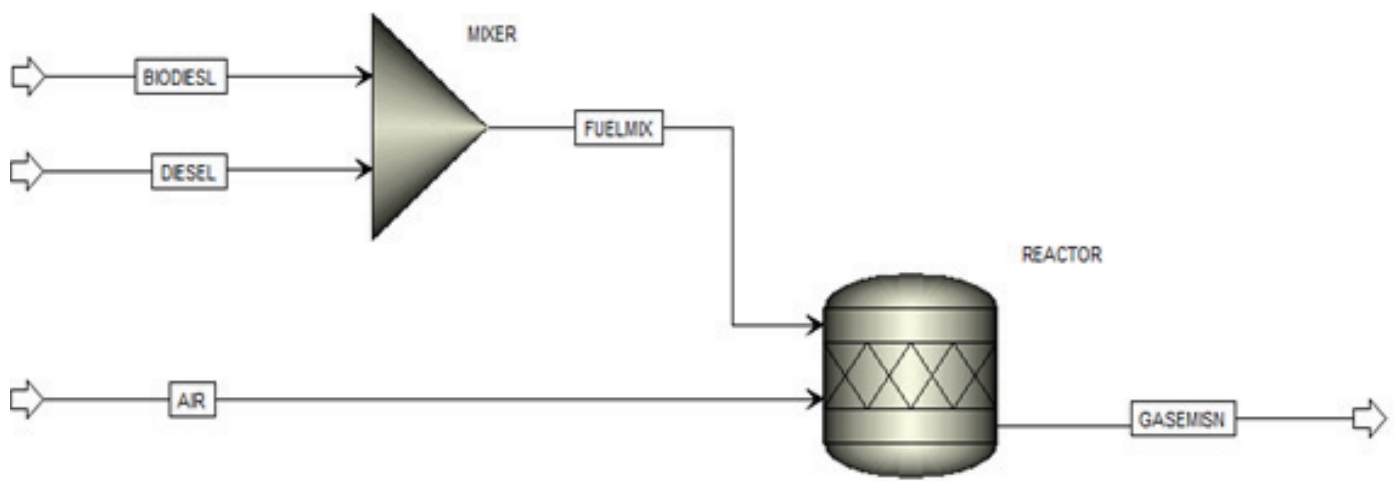

Figura 3. 
Tabla 4. Condiciones de las corrientes de alimentación de las mezclas diesel-biodiesel utilizadas en la simulación

\begin{tabular}{lcccccccc}
\hline \multicolumn{1}{c}{\begin{tabular}{c} 
Flujo másico \\
\multicolumn{1}{c}{ (kg/h) }
\end{tabular}} & Aire & B5 & Aire & B10 & Aire & B20 & Aire & B50 \\
\hline Oleato de Metilo & 0 & $9.70 \mathrm{E}-04$ & 0 & $1.93 \mathrm{E}-03$ & 0 & $3.86 \mathrm{E}-03$ & 0 & $9.65 \mathrm{E}-03$ \\
Linoleato de Metilo & 0 & $1.07 \mathrm{E}-03$ & 0 & $2.14 \mathrm{E}-03$ & 0 & $4.29 \mathrm{E}-03$ & 0 & 0.010715 \\
Ricinoleato de Metilo & 0 & 0.0222027 & 0 & 0.0444055 & 0 & 0.088811 & 0 & 0.2220275 \\
Palmitato de Metilo & 0 & $2.72 \mathrm{E}-04$ & 0 & $5.43 \mathrm{E}-04$ & 0 & $1.09 \mathrm{E}-03$ & 0 & $2.72 \mathrm{E}-03$ \\
Estearato de Metilo & 0 & $2.72 \mathrm{E}-04$ & 0 & $5.43 \mathrm{E}-04$ & 0 & $1.09 \mathrm{E}-03$ & 0 & $2.72 \mathrm{E}-03$ \\
Linolenato de Metilo & 0 & $1.09 \mathrm{E}-04$ & 0 & $2.18 \mathrm{E}-04$ & 0 & $4.36 \mathrm{E}-04$ & 0 & $1.09 \mathrm{E}-03$ \\
Eicoseonato de Metilo & 0 & $1.09 \mathrm{E}-04$ & 0 & $2.18 \mathrm{E}-04$ & 0 & $4.36 \mathrm{E}-04$ & 0 & $1.09 \mathrm{E}-03$ \\
Diesel & 0 & 0.475 & 0 & 0.45 & 0 & 0.4 & 0 & 0.25 \\
Oxígeno & 12.84675 & 0 & 12.11691 & 0 & 12.43662 & 0 & 11.61615 & 0 \\
Nitrógeno & 48.32825 & 0 & 47.81396 & 0 & 46.78538 & 0 & 43.69885 & 0 \\
\hline
\end{tabular}

Tabla 5. Composición de ésteres metílicos de biodiesel de higuerilla

\begin{tabular}{lcc}
\hline \multicolumn{1}{c}{ Éster Metílico } & Fracción $(\%)$ & $\begin{array}{c}\text { Fórmula } \\
\text { condensada }\end{array}$ \\
\hline Ricinoleato de Metilo & 88.811 & $\mathrm{C}_{19} \mathrm{H}_{36} \mathrm{O}_{3}$ \\
Linoleato de Metilo & 4.286 & $\mathrm{C}_{19} \mathrm{H}_{34} \mathrm{O}_{2}$ \\
Oleato de Metilo & 3.860 & $\mathrm{C}_{19} \mathrm{H}_{36} \mathrm{O}_{2}$ \\
Estearato de Metilo & 1.086 & $\mathrm{C}_{19} \mathrm{H}_{38} \mathrm{O}_{2}$ \\
Palmitato de Metilo & 1.086 & $\mathrm{C}_{17} \mathrm{H}_{34} \mathrm{O}_{2}$ \\
Linolenato de Metilo & 0.436 & $\mathrm{C}_{19} \mathrm{H}_{32} \mathrm{O}_{2}$ \\
Eicoseonato de Metilo & 0.436 & $\mathrm{C}_{21} \mathrm{H}_{40} \mathrm{O}_{2}$ \\
\hline
\end{tabular}

Tabla 6. Reacciones químicas del proceso de combustión de los ésteres metílicos y diesel

\begin{tabular}{clll}
\hline Núm. Rx & \multicolumn{1}{c}{ Compuesto } & \multicolumn{2}{c}{ Estequiometría } \\
\hline 1 & Ricinoleato de Metilo & $\mathrm{C}_{19} \mathrm{H}_{36} \mathrm{O}_{3}+26.5 \mathrm{O}_{2}$ & $18 \mathrm{H}_{2} \mathrm{O}+19 \mathrm{CO}_{2}$ \\
2 & Oleato de Metilo & $\mathrm{C}_{19} \mathrm{H}_{36} \mathrm{O}_{2}+27 \mathrm{O}_{2}$ & $18 \mathrm{H}_{2} \mathrm{O}+19 \mathrm{CO}_{2}$ \\
3 & Linoleato de Metilo & $\mathrm{C}_{19} \mathrm{H}_{34} \mathrm{O}_{2}+26.5 \mathrm{O}_{2}$ & $17 \mathrm{H}_{2} \mathrm{O}+19 \mathrm{CO}_{2}$ \\
4 & Estearato de Metilo & $\mathrm{C}_{19} \mathrm{H}_{38} \mathrm{O}_{2}+27.5 \mathrm{O}_{2}$ & $19 \mathrm{H}_{2} \mathrm{O}+19 \mathrm{CO}_{2}$ \\
5 & Palmitato de Metilo & $\mathrm{C}_{17} \mathrm{H}_{34} \mathrm{O}_{2}+24.5 \mathrm{O}_{2}$ & $17 \mathrm{H}_{2} \mathrm{O}+17 \mathrm{CO}_{2}$ \\
6 & Linolenato de Metilo & $\mathrm{C}_{19} \mathrm{H}_{32} \mathrm{O}_{2}+26 \mathrm{O}_{2}$ & $16 \mathrm{H}_{2} \mathrm{O}+19 \mathrm{CO}_{2}$ \\
7 & Eicoseonato de Metilo & $\mathrm{C}_{21} \mathrm{H}_{40} \mathrm{O}_{2}+30 \mathrm{O}_{2}$ & $20 \mathrm{H}_{2} \mathrm{O}+21 \mathrm{CO}_{2}$ \\
8 & Diesel (n-Hexadecano) & $\mathrm{C}_{16} \mathrm{H}_{34}+24.5 \mathrm{O}_{2}$ & $17 \mathrm{H}_{2} \mathrm{O}+16 \mathrm{CO}_{2}$ \\
\hline
\end{tabular}

\section{Resultados y discusiones}

Una vez ingresados los datos en el simulador, se obtuvieron los poderes caloríficos para cada uno de los ésteres metílicos que conforman el biodiesel obtenido a partir de la higuerilla como se muestra en la tabla 7. Es de esperarse que el PCI del biodiesel, sea lo más aproximado al del ricinoleato de metilo, debido a que este último es el compuesto que se encuentra en mayor porcentaje de la composición con $88.81 \%$. 
Tabla 7. PCl de los ésteres metílicos de aceite de higuerilla

\begin{tabular}{lc}
\hline \multicolumn{1}{c}{ Ésteres Metílicos } & PCI $(\mathrm{MJ} / \mathrm{kg})$ \\
\hline Oleato de Metilo & 37.43 \\
Linoleato de Metilo & 40.13 \\
Ricinoleato de Metilo & 38.16 \\
Palmitato de Metilo & 39.12 \\
Estearato de Metilo & 42.82 \\
Linolenato de Metilo & 37.51 \\
Eicoseonato de Metilo & 40.84 \\
\hline
\end{tabular}

Los resultados reflejan que el B100 presenta un PCI menor al del diesel, con una variación del $12.85 \%$, por lo que el PCI de las diferentes mezclas diesel-biodiesel tienen una tendencia a disminuir conforme se incrementa la cantidad de biodiesel, tal como se puede observar en la figura 4. Dichos resultados son congruentes con los datos reportados por diferentes autores (Iscan y Aydin, 2012; Canacki y Gerpen, 1999; Vyas et al., 2010; Nielsen et al., 2008; Boey et al., 2009).

Las variaciones porcentuales del poder calorífico para las mezclas restantes con respecto al diesel, B50, B20, B10 y B5 fueron de $6.42 \%, 2.57 \%, 1.27 \%$ y $0.54 \%$, respectivamente.

La disminución del poder calorífico tanto del biodiesel como sus mezclas, representa una desventaja importante comparado con el diesel, puesto que implica repercusiones directas que se reflejarían en un descenso de la potencia y torque del motor, así como un incremento en el consumo de combustible (Benavides et al., 2007).

El NREL (National of Renewable Energy Laboratory, USA) especifica los valores de PCI del diesel y biodiesel obtenido a partir de diversas fuentes de materia prima como son: soya, canola, manteca, grasas animales no comestibles y grasas amarillas de alta y baja acidez, ilustrados en la tabla 8.

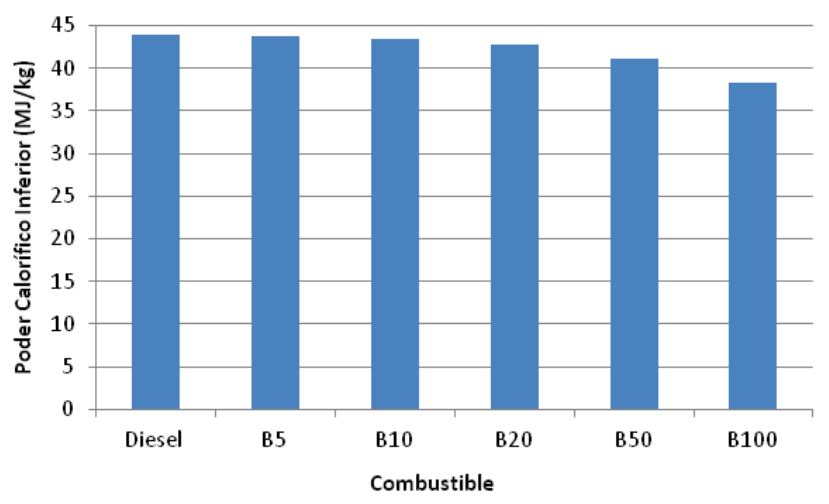

Figura 4. Poder calorífico de los combustibles
Tabla 8. PCl de diesel y tipos de biodiesel a partir de diversas materias primas (Adaptado de NREL)

\begin{tabular}{lc}
\hline \multicolumn{1}{c}{ Combustibles } & PCI [MJ/kg] \\
\hline Diesel Núm. 2 & 42.57 \\
Biodiesel de soya & 37.08 \\
Biodiesel de canola & 36.89 \\
Biodiesel de manteca & 36.85 \\
Biodiesel de cebo comestible & 36.94 \\
Biodiesel de cebo no comestible & 36.85 \\
Biodiesel de grasa amarilla de baja acidez & 36.95 \\
Biodiesel de grasa amarilla de alta acidez & 36.54 \\
\hline
\end{tabular}

Como se puede observar, el PCI del biodiesel de higuerilla obtenido a partir de la simulación, estimado en $38.28 \mathrm{MJ} / \mathrm{kg}$, se posiciona por encima de los valores referenciados por NREL. De acuerdo con Benavides $e t$ al., (2007), el PCI medido para el biodiesel de higuerilla es de $37.52 \mathrm{MJ} / \mathrm{kg}$. Esto presenta una diferencia porcentual de $1.02 \%$ del PCI simulado con respecto al experimental.

\section{Emisiones}

Una de las ventajas más importantes que tiene el biodiesel, es el beneficio ambiental, pues su utilización representa una oportunidad para contribuir en la mitigación de emisiones de $\mathrm{CO}_{2}$. Ello ayudaría al mejoramiento de la calidad de aire y salud pública en lo que a enfermedades crónicas respiratorias se refiere.

Los resultados contenidos en la tabla 9 indican una disminución de emisiones de $\mathrm{CO}_{2}$ asociada con el aumento de la concentración de biodiesel.

La variación porcentual de emisiones de $\mathrm{CO}_{2}$ obtenida del B5 al B100 presentó una reducción que va desde $0.7 \%$ hasta $13.5 \%$, respectivamente, como se ilustra en la figura 5.

Tabla 9. Emisiones de diesel, biodiesel y mezclas a $144.5^{\circ} \mathrm{C}$ y $4.6 \mathrm{MPa}$

\begin{tabular}{ccccccc}
\hline $\begin{array}{c}\text { Flujo } \\
\text { másico } \\
(\mathrm{kg} / \mathrm{h})\end{array}$ & Diesel & B5 & B10 & B20 & B50 & B100 \\
\hline $\mathrm{H}_{2} \mathrm{O}$ & 0.2367 & 0.234 & 0.2313 & 0.2258 & 0.2095 & 0.1824 \\
$\mathrm{O}_{2}$ & 12.3778 & 12.2473 & 12.1169 & 11.8562 & 11.0739 & 9.7701 \\
$\mathrm{CO}_{2}$ & 0.5442 & 0.5405 & 0.5369 & 0.5296 & 0.5077 & 0.4712 \\
$\mathrm{~N}_{2}$ & 48.8433 & 48.3283 & 47.8140 & 46.7854 & 43.6989 & 38.5544 \\
\hline
\end{tabular}




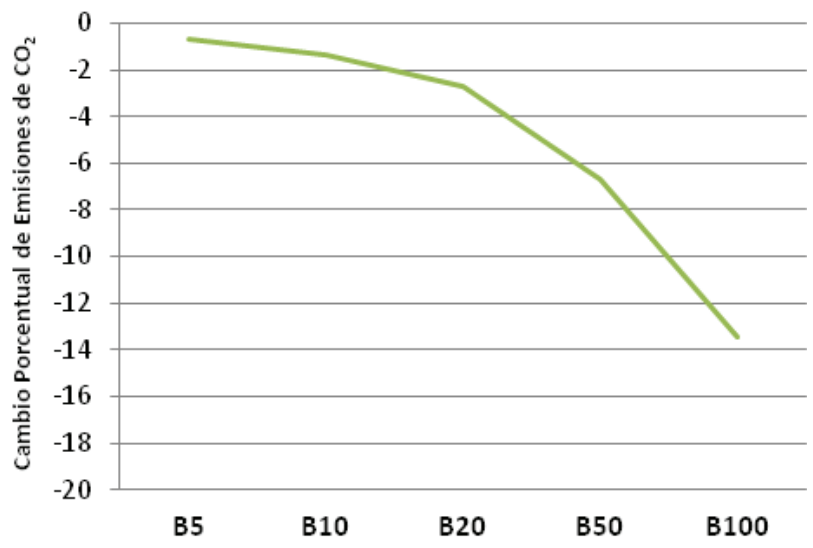

Figura 5. Variación porcentual de emisiones de $\mathrm{CO}_{2}$ vs concentración de biodiesel

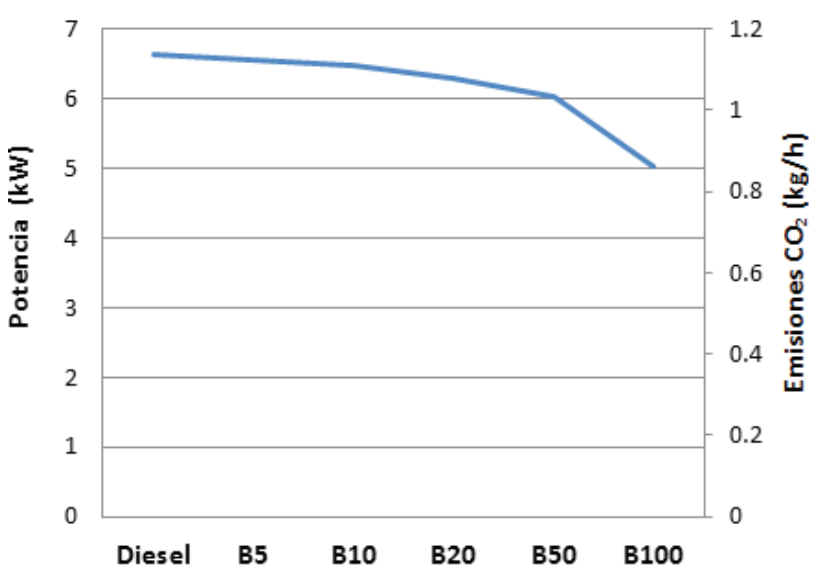

Figura 6. Comportamiento de la potencia y emisiones de $\mathrm{CO}_{2}$ en relación al tipo de combustible

La figura 6 muestra la relación entre potencia y emisiones de $\mathrm{CO}_{2}$ en función de la concentración de biodiesel. Se puede observar que al incrementar la concentración de biodiesel disminuye la potencia generada y al mismo tiempo se reducen las emisiones de $\mathrm{CO}_{2}$.

\section{Conclusiones}

El uso del simulador ASPEN ${ }^{\circledR}$ para recrear un proceso de combustión, utilizando mezclas de diesel-biodiesel de higuerilla, con una composición de ésteres metílicos determinada, proporcionó información importante sobre este proceso. Considerando al biodiesel como combustible permitió:

a) Determinar el poder calorífico del biodiesel y sus mezclas con diesel, utilizando una composición más apegada a la realidad. b) Confirmar que el incremento de la concentración de biodiesel en la mezcla ocasiona una disminución del PCI.

c) Estimar las emisiones de $\mathrm{CO}_{2}$ generadas por la combustión del diesel, biodiesel y mezclas, para realizar una comparación entre los diferentes combustibles.

d) Confirmar a través de los resultados, la diminución de las emisiones de $\mathrm{CO}_{2}$ que implica la sustitución del diesel por biodiesel. Sin embargo, a primera vista puede que no parezca una disminución sustancial, pero no hay que olvidar que el biodiesel, al provenir de fuentes renovables como la biomasa, deja de aportar a la atmósfera una gran fracción del $\mathrm{CO}_{2}$, que fue utilizado por las plantas oleaginosas, para su desarrollo.

Por último se recomienda la realización experimental de mediciones de los PCI para comparar y validar los resultados simulados. Asimismo, cuantificar las emisiones contaminantes de motores operando con diesel y biodiesel para validar y comparar los resultados experimentales con los simulados.

\section{Agradecimientos}

Se agradece el apoyo recibido de CONACYT y el Instituto de Ingeniería de la Universidad Autónoma de Baja California para la realización del presente trabajo.

\section{Referencias}

Altin R., Cetinkaya S., Yucesu H.S. The Potential of Using Vegetable Oil Fuels as Fuel for Diesel Engines. Energy Conversion and Management [en línea] volumen 42 (número 5), 2001 [fecha de consulta: 24 de febrero de 2013]. Disponible en: http://www. sciencedirect.com/science/article/pii/S0196890400000807.

Benavides A., Benjumea P., Pashova V. El biodiesel de aceite de higuerilla como combustible alternativo para motores Diesel. Dyna [en línea] volumen 74 (número 153), 2007 [fecha de consulta: 14 de enero de 2013]. Disponible en: http://dyna.unalmed.edu.co/ediciones/153/articulos/AB230806/AB230806.pdf.

Boey P., Maniam G., Hamid S. Biodiesel from Adsorbed Waste Oil on Spent Bleaching Clay using $\mathrm{CaO}$ as a Heterogeneous Catalyst. European Journal of Scientific Research [en línea] volumen 33 (número 2), 2009 [fecha de consulta: 25 de febrero de 2013]. Disponible en: http://connection.ebscohost.com/c/articles/ 44090135/biodiesel-from-adsorbed-waste-oil-spent-bleaching-clay-using-cao-as-heterogeneous-catalyst.

Canacki M. Combustion Characteristics of a Turbocharged DI Compression Ignition Engine Fueled with Petroleum Diesel Fuels and Biodiesel. Bioresource Technology [en línea] volumen 98 (número 6), 2007 [fecha de consulta: 20 de febrero de 2013]. 
Disponible en: http://www.sciencedirect.com/science/article/ pii/S0960852406002306.

Canacki M., Gerpen J.V. Biodiesel Production Via Acid Catalysis. American Society of Agricultural Engineers [en línea] volumen 42 (número 5), 1999 [fecha de consulta: 20 de febrero de 2013]. Disponible en: http://elibrary.asabe.org/abstract.asp?aid=132 $85 \& \mathrm{t}=2 \&$ redir $=\&$ redirType $=$.

Carlson E.C. Don't Gamble with Physical Properties for Simulations. Chemical Engineering Progress [en línea], 1996 [fecha de consulta 20 de diciembre de 2012]. Disponible en: http:// www.eq.uc.pt/ nuno/eps/old/2011-12/aula08/Carlson\%20 (1996).pdf.

Cedeño L., Martínez E., Gómez M., Pegraza F. Desulfuración de Organoazufrados presentes en diesel por oxidación y extracción, Parte 1 Catalizadores de Cobre Soportados. Revista Mexicana de Ingeniería Química [en línea], volúmen 4 (número 3), 2005 [fecha de consulta 7 de diciembre de 2012]. Disponible en: http://www.redalyc.org/articulo.oa?id=62040304.

Graboski M.S, McCormick R.L. Combustion of Fat and Vegetable Oil Derived Fuels in Diesel Engines. Progress in Energy and Combustion Science [en línea] volumen 24 (número 2), 1998 [fecha de consulta: 15 de febrero de 2013]. Disponible en: http:// www.sciencedirect.com/science/article/pii/S0360128597000348.

Heywood J.B. Internal Combustion Engine Fundamentals, McGrawHill, series in Mechanical Engineering, Nueva York, 1988.

INIFAP. Informe de autoevaluación del director general del INIFAP correspondiente al ejercicio 2009 [en línea] 2009 [fecha de consulta: 11 de abril de 2013]. Disponible en: http://www.encuentra.gob.mx/resultsAPF.html?q=higuerilla\%20inifap\&clie $\mathrm{nt}=$ inifap\&ts $=$ all\&geo $=0$.

Iscan B. y Aydin H. Improving the Usability of Vegetable Oil as Fuel in a Low Heat Rejection Diesel Engine. Fuel Processing Technology [en linea] volumen 98 (números 59-64), 2012 [fecha de consulta: 18 de febrero del 2013]. Disponible en: http://www. sciencedirect.com/science/article/pii/S0378382012000483\#.

Lin Y.C, Lee W.J, Hou H.C. PAH Emissions and Energy Efficiency of Palm Biodiesel Blends Fueled on Diesel Generator. Atmospheric Environment [en línea] volumen 40 (número 21), 2006 [fecha de consulta: 22 de febrero de 2013]. Disponible en: http://www. sciencedirect.com/science/article/pii/S1352231006002330.

National Renewable Energy Laboratory. Biodiesel Handing and Use Guide [en línea] Technical Report TP-540-43672, 4a ed., Springfield, VA, 2009 [fecha de consulta: 15 de noviembre de 2012]. Disponible en: http://www.nrcs.usda.gov/Internet/FSE_DOCUMENTS/stelprdb1045416.pdf.

Nielsen P., Brask J., Fjerbaek L. Enzymatic Biodiesel Production. Technical and Economical consideration. European Journal of
Lipid Science and Technology [en línea] volumen 110 (número 8), 2008 [fecha de consulta: 2 de marzo de 2013]. Disponible en: http://onlinelibrary.wiley.com/doi/10.1002/ejlt.200800064/ abstract.

Rakopoulos C.D, Antonopoulos K.A et al. Comparative Performance and Emissions Study of a Direct Injection Diesel Engine Using Blends of Diesel Fuel with Vegetable Oils or Biodiesels of Various Origins. Energy Conversion and Management [en línea] volumen 47 (números 18-19), 2006 [fecha de consulta: 22 de febrero de 2013]. Disponible en: http://www. sciencedirect.com/science/article/pii/S0196890406000379.

Refaat A. Biodiesel Production Using Solid Metal Oxide Catalysts. International Journal of Environment Science Technology [en línea] volumen 8 (número 1), 2011 [fecha de consulta: 24 de febrero de 2013]. Disponible en: http://www.bioline.org.br/ pdf?st11020.

Scarpa A. y Guerci A. Various Uses of the Castor Oil Plant (Ricinus Communis L.) Journal of Ethnopharmacology [en línea] volumen 5 (número 2), 1982 [fecha de consulta: 5 de diciembre de 2012]. Disponible en: http://www.sciencedirect.com/science/ article/pii/0378874182900381.

SENER. Balance Nacional de Energía 2009 [en línea], México D.F., 2010 [fecha de consulta 14 de mayo de 2012]. Disponible en: http://www.sener.gob.mx/res/1791/Balance_Nacional_2009.pdf.

Vyas A., Berma J., Subrahmanyam N. A Review on FAME Production Processes. Fuel [en línea] volumen 89 (número 1), 2010 [fecha de consulta: 19 de enero de 2013]. Disponible en: http:// www.sciencedirect.com/science/article/pii/S0016236109003755.

Workshop Manual Diesel Engine Kubota for Engine Models: Z400-B. Z430-B. D600-B. D640-B V800-B, Kubota.

\section{Este artículo se cita:}

\section{Citación estilo Chicago}

Pérez-Sánchez, Armando, Gisela Montero-Alpírez, José Ramón Ayala-Bautista, Marcos Alberto Coronado-Ortega, Conrado García-González, Héctor Enrique Campbell-Ramírez. Simulación en Aspen de la combustión de mezclas diesel-biodiesel. Ingeniería Investigación y Tecnología, XVI, 01 (2015): 83-92.

\section{Citación estilo ISO 690}

Pérez-Sánchez A., Montero-Alpírez G., Ayala-Bautista J.R., Coronado-Ortega M.A., García-González C., Campbell-Ramírez H.E. Simulación en Aspen de la combustión de mezclas diesel-biodiesel. Ingeniería Investigación y Tecnología, volumen XVI (número 1), enero-marzo 2015: 83-92. 


\section{Semblanza de los autores}

Armando Pérez-Sánchez. Estudiante de doctorado en ingeniería en el Instituto de Ingeniería de la Universidad Autónoma de Baja California (UABC). Maestro en ingeniería por el Instituto de Ingeniería de la UABC (2012). Ingeniero mecánico por la UABC (2008). Desde 2011 es docente en la Facultad de Ingeniería de la UABC, en las carreras de ingeniería industrial e ingeniería aeroespacial. Coautor de capítulos de libro, artículos en revistas indexadas y cuenta con dos solicitudes de patente. Ha participado como ponente en congresos nacionales e internacionales.

Gisela Montero-Alpírez. Ingeniera química por la Universidad Nacional Autónoma de México (UNAM). Doctora en ciencias químicas por la UNAM. Maestra en ingeniería termodinámica por la UABC. Investigadora del Instituto de Ingeniería de la UABC. Sus temas de interés son biocombustibles, análisis de exergia de procesos, simulación de procesos y extracción de aceites esenciales. Autora de varios artículos en revistas indexadas, reportes de investigación y cuenta con dos solicitudes de patente. Ha editado dos libros sobre biodiesel y tiene publicados varios capítulos de libro sobre energía y biocombustibles. Miembro del SNI Nivel 1.

José Ramón Ayala-Bautista. Ingeniero químico por el Instituto Tecnológico de Mexicali. Premio a la excelencia académica 2010, otorgado por la Asociación Nacional de Facultades y Escuelas de Ingeniería. Trabajó para Skyworks Solutions de México en proyectos de reducción de residuos y su manejo. Maestro en ingeniería por la UABC (mención honorífica). Es docente de licenciatura en la misma universidad y ha contribuido con el diseño de cartas descriptivas para las materias de transferencia de masa y planeación energética.

Marcos Alberto Coronado-Ortega. Ingeniero industrial, por la UABC. Doctor y maestro en ingeniería, área de química-energía por el Instituto de Ingeniería de la UABC (mérito escolar en maestría y mención honorífica en ambos). Autor de diversos artículos y capítulos de libro sobre energía y biocombustibles. Ponente en congresos nacionales e internacionales. Investigador del laboratorio de biocombustibles del Instituto de Ingeniería, UABC. Profesor en la Facultad de Ingeniería de la misma universidad. Miembro del SNI, nivel candidato.

Conrado García-González. Ingeniero químico, por el Instituto Tecnológico de Mexicali (ITM), especializado en medio ambiente. Doctor en ingeniería (mención honorifica, área de química-energía) por el Instituto de Ingeniería de la UABC. Ha escrito varios artículos y capítulos de libros relacionados con la energía de la biomasa y biocombustibles. Ha participado como ponente en congresos nacionales e internacionales. Es profesor-investigador en proyectos de biomasa, optimización de procesos para la obtención de biocombustibles e hidrógeno, y es coordinador de proyectos de investigación en el Departamento de Química y Bioquímica del ITM. Miembro del SNI a nivel candidato.

Héctor Enrique Campbell-Ramírez. Investigador en el Instituto de Ingeniería, UABC. Ingeniero químico por la Universidad de Sonora. Maestro en ingeniería termodinámica y doctor en ingeniería por el Instituto de Ingeniería, UABC. Especialidad en ahorro y uso eficiente de energía, planificación energética, ingeniería de costos, mecánica de fluidos y transferencia de calor. Tiene 49 proyectos de investigación, la mayoría relacionados con el impacto social y económico de los recursos energéticos; 27 publicaciones y 53 ponencias en congresos en México, EU, Nueva Zelanda, Uruguay, El Salvador y Argentina. Profesor de 30 diferentes cursos de licenciatura y postgrado. 